\title{
Cathepsin D is involved in the oxygen and glucose deprivation/reperfusion-induced apoptosis of astrocytes
}

\author{
JIANLIN LIU, LIN YANG, HONGYAN TIAN and QIANG MA \\ Department of Neurology, The First Affiliated Hospital, School of Medicine, \\ Xi'an Jiaotong University, Xi'an, Shaanxi 710061, P.R. China
}

Received November 24, 2015; Accepted July 12, 2016

DOI: $10.3892 / \mathrm{ijmm} .2016 .2709$

\begin{abstract}
The lysosome and its associated protein cathepsin D (Cat D) play critical roles in the pathological process of secondary damage following ischemia/reperfusion (I/R) injury. However, the roles of Cat D in I/R-exposed astrocytesremain unclear. In this study, we determined the roles of Cat D in the oxygen-glucose deprivation/reperfusion (OGD/R)-induced apoptosis of astrocytes as well as the underlying mechanisms. We found that OGD/R markedly increased cell apoptosis and the production of inflammatory cytokines, namely IL-6, tumor necrosis factor (TNF)- $\alpha$ and FasL in a reperfusion time-dependent manner and their elevation peaked at $24 \mathrm{~h}$ after reperfusion. Moreover, the cytosolic Cat D level and Cat D activity was significantly upregulated in response to OGD/R exposure. Furthermore, OGD/R exposure gradually disrupted the innate acidic conditions of the lysosome. Exogenous TNF- $\alpha$ and FasL administration elevated cytosolic Cat D levels and cell apoptosis whereas TNFR1 and Fas inhibition significantly reversed these effects induced by OGD/R. Cat D overexpression enhanced cell apoptosis and the levels of apoptogenic proteins, including Bax and caspase-3, whereas Cat D siRNA transfection had an inhibitory effect on cell apoptosis and the expression of proapoptotic proteins. In addition, we observed that Cat D upregulation disrupted mitochondrial membrane potential and induced the production of reactive oxygen species. In conclusion, OGD/R injury induced the production of TNF- $\alpha$, IL-6 and FasL which promoted lysosomal dysfunction and Cat D leakage into the cytoplasm. This eventually resulted in caspase-dependent apoptosis, mitochondrial membrane potential loss and oxidative stress in astrocytes.
\end{abstract}

Correspondence to: Dr Jianlin Liu, Department of Neurology, The First Affiliated Hospital, School of Medicine, Xi'an Jiaotong University, 277 Yanta West Road, Xi'an, Shaanxi 710061, P.R. China E-mail: jlliu@mail.xjtu.edu.cn

Key words: cathepsin D, oxygen-glucose deprivation/reperfusion, astrocyte, lysosome, tumor necrosis factor- $\alpha$, FasL

\section{Introduction}

Stroke ranks among the most lethal cerebrovascular diseases worldwide and is associated with high morbidity and long-term disability, which may cause irreversible brain damage and loss of neuronal function (1). Brain ischemia, characterized by the occlusion of blood vessels and deprivation of energy and nutrition, leads to serious neuronal injury and neurodegeneration, and further to learning and memory impairment (2). Following brain ischemia/reperfusion (I/R) injury, proinflammatory cytokines, such as interleukin (IL)-1 $\beta$, IL-6 and tumor necrosis factor (TNF)- $\alpha$, are immediately produced and secreted after the onset of cerebral ischemia, eventually contributing to the pathogenesis and exacerbation of brain tissue damage (3).

Astrocytes are one type of glial cell in the mammalian central nervous system (CNS), and the most numerous cell type of the brain (4). Under physiologic conditions, astrocytes facilitate neuronal homeostasis in the CNS whereas under pathologic conditions astrocytes promote neuronal demise (5). In the context of cerebral ischemia, all of the star-type cells, microglia as well as endothelial cells, may produce cellular factors $(6,7)$.

Lysosomes consist of more than 50 acid hydrolases and 120 membrane proteins, which participate in lysosomal digestion or the maintenance of lysosomal integrity and the regulation of lysosomal trafficking, fusion and intralysosomal $\mathrm{pH}(8)$. Lysosomal enzymes, including cathepsins (Cats) (Cat B, D, K, $\mathrm{L}$ and so on) and some lipid hydrolases, when released from the ruptured lysosomal membrane, may cause the destruction of cellular components and even lead to cell death (9). It has been demonstrated that Cat B and D markedly increase in primary astrocytes after oxygen-glucose deprivation (OGD) and this is accompanied by the apoptosis of astrocytes (10). Moreover, Cat B and L activation after brain ischemia triggered the tBid-mediated death of astrocytes following brain $\mathrm{I} / \mathrm{R}$ in vivo (11). Accumulating evidence has demonstrated that the lysosome and its associated protein Cat D play critical roles in the pathological process of secondary damage following I/R damage (12). However, the roles of Cat D during the exposure of astrocytes to $\mathrm{I} / \mathrm{R}$ remain unclear. In the present study, we examined the effects of inflammatory molecules on the expression and activation of Cat D as well as the role of Cat D in the OGD-induced death of astrocytes and the underlying mechanism, in order to identify a novel strategy for the treatment of brain damage following I/R injury. 


\section{Materials and methods}

Astrocyte culture and OGD. Astrocytes were isolated from the cerebral cortex of BALB/c mice $(n=5)$ on postnatal days $1-3$ according to previously described protocols (13). All the mice were obtained from the animal center of Xi'an Jiaotong University (Xi'an, China). Briefly, all mice received general anesthesia with isoflurane inhalation prior to sacrifice in order to minimize their suffering. Ethics approval was obtained from the Ethics Committee of Xi'an Jiaotong University. Cortical hemispheres were dissociated with $0.25 \%$ trypsin in PBS. Following filtration, the dissociated cells were placed on polyL-lysine-coated plastic flasks and maintained in Dulbecco's modified Eagle's medium (DMEM)/nutrient mixture F-12 supplemented with $10 \%$ fetal bovine serum (Gibco BRL, Gaithersburg, MD, USA), and cultivated at $37^{\circ} \mathrm{C}$ in a humidified incubator in an atmosphere of $95 \%$ air and $5 \% \mathrm{CO}_{2}$ (Thermo Forma, Marietta, OH, USA). The cells were maintained in complete culture medium for 7-8 days and the culture medium was changed every three days. Astrocyte cultures were passaged twice and the purity of astrocyte cultures consisted of at least 95\% astrocytes as determined by immunostaining against astrocyte marker glial fibrillary acidic protein (GFAP, 1:200; Cell Signaling Technology, Danvers, MA, USA). These cells were used in subsequent experiments.

With regard to the establishment of an in vitro model of OGD using astrocytes, the standard culture medium was refreshed using a glucose-free DMEM buffer (Gibco, Rockville, MD, USA), and the cells were then placed in a hypoxic humidified incubator flushed with a gas mixture of $93 \% \mathrm{~N}_{2} / 5 \% \mathrm{CO}_{2} / 2 \% \mathrm{O}_{2}$ (Thermo Forma) as previously described (14). Four hours later, the cells were cultured in standard medium containing glucose under conditions of normoxia for reoxygenation for an additional $48 \mathrm{~h}$. The control group was comprised of naïve astrocytes cultured under normal conditions and the OGD group was defined as cells exposed to OGD without reperfusion. TNF- $\alpha /$ FasL $(20 \mathrm{ng} / \mathrm{ml})$ were added to naïve astrocytes and incubated for $24 \mathrm{~h}$ for further experiments, and $1 \mu \mathrm{g} / \mathrm{ml}$ anti-Fas (anti-CD95-ZB4; Millipore, Billerica, MA, USA) and anti-tumor necrosis factor receptor 1 (TNFR1; R\&D Systems, Minneapolis, MN, USA) were added to astrocyte cultures $3 \mathrm{~h}$ prior to the OGD/reperfusion (OGD/R) procedure.

Preparation and transfection of small interfering RNA(siRNA). Cat $\mathrm{D}$ knockdown was accomplished by transfecting astrocytes with siRNA. Cat D and control siRNA were synthesized by Wolsen Biological Co., Ltd. (Xi'an, China). Cells $\left(3 \times 10^{5}\right)$ were transfected with $1 \mu \mathrm{g}$ siRNA using $2.5 \mu \mathrm{g}$ Lipofectamine 2000 reagent (Invitrogen, Carlsbad, CA, USA). Cat D knockdown was analyzed $48 \mathrm{~h}$ after transfection by western blot analysis.

Transient transfection of Cat D plasmids. A pCMV-SPORT6 plasmid containing the Cat $\mathrm{D}$ encoding region was purchased from Thermo Fisher Scientific (Ottawa, ON, Canada). Cultured astrocytes were transfected with the Cat D-overexpressing plasmid or a control plasmid using Lipofectamine 2000 according to the manufacturer's instructions. The overexpression of Cat $\mathrm{D}$ was analyzed $48 \mathrm{~h}$ after transfection by western blot analysis. The cells harboring Cat D or control vectors were then subjected to OGD/R exposure.
MTT assay. The viability of the primary astrocytes subjected to OGD/R or not were determined by MTT assay. In brief, the cells were seeded in 96-well plates at a density of $1 \times 10^{4}$ cells in $300 \mu \mathrm{l}$ medium. The control astrocytes were incubated in standard condition and the OGD/R-subjected cells were exposed to $4 \mathrm{~h}$ of OGD followed by reperfusion. At each time point, $50 \mu \mathrm{l}$ of MTT solution (Sigma-Aldrich, St Louis, MO, USA) dissolved in culture medium at a final concentration of $0.5 \mathrm{mmol} / 1$ were added to each well and the plates were incubated at $37^{\circ} \mathrm{C}$ for a further $4 \mathrm{~h}$. Subsequently, the culture medium was discarded and $300 \mu \mathrm{l}$ DMSO (Sigma-Aldrich) was then added to each well, followed by shaking for 20 min to solubilize the MTT tetrazolium crystal. Finally, the absorbance was measured at $570 \mathrm{~nm}$ using a Benchmark Plus microplate reader (Model 550; Bio-Rad Laboratories, Inc., Hercules, CA, USA).

Cell apoptosis. Cells were incubated with Annexin V-FITC and propidium iodide (BD Biosciences, Franklin Lakes, NJ, USA) for $10 \mathrm{~min}$ on ice. Apoptosis was analyzed using a FACSCalibur ${ }^{\circledR}$ flow cytometer (BD Biosciences). Data were processed using FlowJo software.

Western blot analysis. The astrocytes were harvested and lysed in lysis buffer. The extracted proteins (30-50 $\mu \mathrm{g}$ ) were separated on SDS-PAGE gels and transferred to PVDF membranes. After blocking with 5\% skimmed milk, the blots were incubated overnight at $4^{\circ} \mathrm{C}$ with antibodies against cleaved caspase-3 (Asp175; \#9661; Cell Signaling Technology), Bax (sc-7480) and $\beta$-actin (sc-47778) (Santa Cruz Biotechnology, Santa Cruz, CA, USA). Total cytosolic and mitochondrial protein extraction were performed as previously described (7). The extracted cytosolic Cat B (ab58802), D (ab6313) and L (ab58991) (Abcam, Cambridge, UK) were detected. Finally, the immunoreactive bands were visualized using an ECL detection system. The immunoreactive bands were visualized using an ECL detection system (Amersham Pharmacia Biotech, Uppsala, Sweden). Signals were quantified by a grayscale scanner (GeneGnome XRQ; Syngene Corp., Cambridge, UK).

Reverse transcription-quantitative PCR (RT-qPCR). Total RNA was extracted from the treated astrocytes using TRIzol reagent (Invitrogen) and was reverse transcribed into cDNA using the M-MLV Reverse Transcriptase kit (Invitrogen) according to the manufacturer's instructions. Quantitative PCR (qPCR) was run on a Bio-Rad CFX manager using SYBR Premix Ex Taq ${ }^{\mathrm{TM}}$ (Takara). The PCR primer sequences used were as follows: IL-6 sense, 5'-CTG CAA GAG ACT TCC ATC CAG TT-3' and antisense, 5'-AGG GAA GGC CGT GGT TGT-3'; TNF- $\alpha$ sense, 5'-ACG TGC AGC TAC TGC ATG TGA-3' and antisense, 5'-AGA AGG AAC ACG TTG TCA GCG-3'; FasL sense, 5'-AGC CCG TGA ATT ACC CAT GTC-3' and antisense, 5'-TGC TGG GGT TGG CTA TTT GCT-3'; GAPDH sense, 5'-AGC AGT CCC GTA CAC TGG CAA AC-3' and antisense, 5'-TCT CCT GTA AAT GTA GTG GTG TCT-3'. PCR was performed on an iCycler iQ (Bio-Rad Laboratories, Inc.) beginning at $95^{\circ} \mathrm{C}$ for $20 \mathrm{sec}$, followed by 40 cycles: at $95^{\circ} \mathrm{C}$ for $1 \mathrm{sec}$, and at $60^{\circ} \mathrm{C}$ for $20 \mathrm{sec}$. Gene expression was determined by the $2^{-\Delta \Delta \mathrm{Ct}}$ method.

Determination of Cat D activity. A Cat D activity assay kit (Abcam) was used to determine the activity of Cat D following 
A
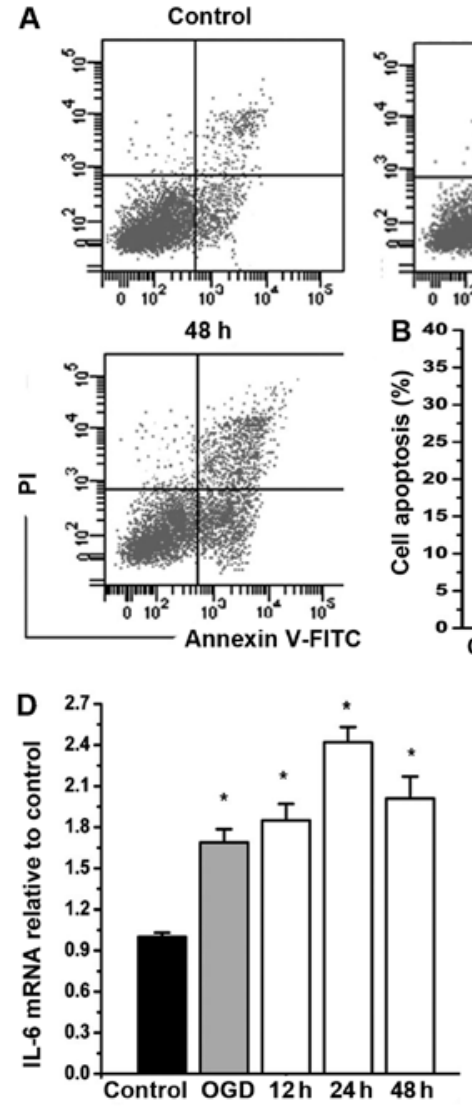

OGD

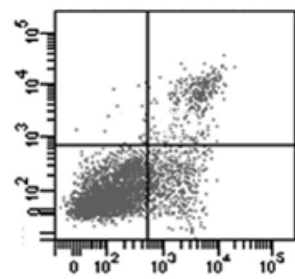

B. $^{30}$ ].

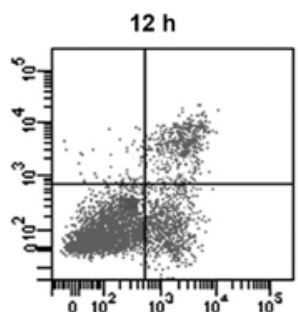

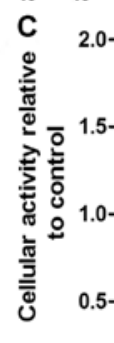

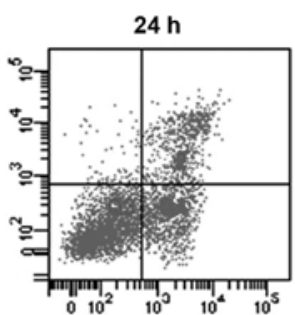

-o. Control

廿 OGD/R

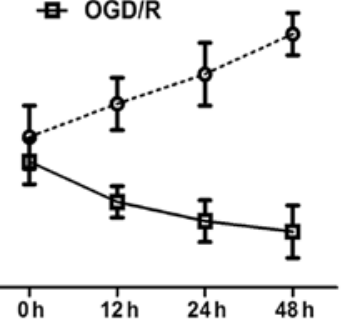

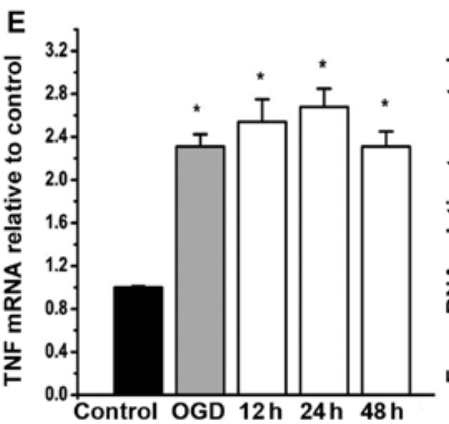

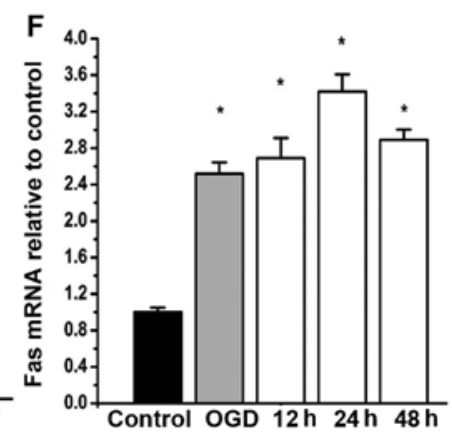

Figure 1. Oxygen-glucose deprivation/reperfusion (OGD/R) injury induces the apoptosis of astrocytes and an inflammatory response. Murine astrocytes were isolated and subjected to OGD for $4 \mathrm{~h}$ followed by reperfusion treatment over a time period ranging from 0 to $48 \mathrm{~h}$. (A and B) The apoptosis of astrocytes was examined and quantified using flow cytometry. (C) Cell viability was evaluated using the MTT assay at the indicated time points within 48 h of reperfusion. RT-PCR analysis of the mRNA levels of (D) interleukin (IL)-6, (E) tumor necrosis factor (TNF)- $\alpha$ and (F) FasL in astrocytes after exposure to OGD/R treatments for various periods of time. ${ }^{*} \mathrm{P}<0.05$, vs. control. Each experiment was performed in triplicate.

OGD/R exposure. The substrate cleaved by Cat D in cell lysates releases fluorescence which is captured by a fluorescence plate reader at $\mathrm{Ex} / \mathrm{Em}=328 / 460 \mathrm{~nm}$.

LysoTracker assay. Following incubation with $1 \mu \mathrm{M}$ LysoTracker Red dye (Invitrogen) for $15 \mathrm{~min}$, the astrocytes were trypsinized and resuspended in PBS for FACS analysis (FACSCanto II; BD Biosciences, San Jose, CA, USA). Cyflogic software (CyFlo Ltd., Turku, Finland) was used to analyze 20,000 events/run.

Evaluation of mitochondrial membrane potential $\left(\Delta \Psi_{m}\right)$ and reactive oxygen species (ROS) production. Following exposure to OGD/R for $24 \mathrm{~h}$, the astrocytes were washed with PBS and evaluated for time-dependent changes in $\Delta \Psi_{\mathrm{m}}$ by resuspension in freshly prepared JC-1-containing (Invitrogen) medium, which was followed by $30 \mathrm{~min}$ of cultivation in the dark at room temperature. Fluorescence intensity was measured with excitation at $490 \mathrm{~nm}$ and emission at 530 and $590 \mathrm{~nm}$ using a Bio-Rad microplate reader (Model 680; Bio-Rad Laboratories, Inc.). The ratio between green and red fluorescence provides an estimate of $\Delta \Psi_{\mathrm{m}}$ that is independent of mitochondrial mass. For the ROS assay, treated cells were exposed to DCFH-DA for $15 \mathrm{~min}$ at $37^{\circ} \mathrm{C}$. Fluorescence excitation and emission wavelengths were set at 480 and $530 \mathrm{~nm}$, respectively, using a Bio-Rad microplate reader.
Statistical analysis. Values are expressed as the means \pm SEM. Data were analyzed by one-way ANOVA followed by Fisher's LSD test and the Bonferroni test using SPSS 13.0 software. $\mathrm{P}<0.05$ was considered to indicate a statistically significant difference. All experiments were performed at least in triplicate.

\section{Results}

OGD/R injury triggers the production of inflammatory mediators and the apoptosis of astrocytes. In order to examine the destructive effects of OGD/R injury on astrocytes, cell apoptosis and the expression of inflammatory factors, namely IL-6, TNF- $\alpha$ and FasL, were monitored within $48 \mathrm{~h}$ of reperfusion following $4 \mathrm{~h}$ of OGD. Flow cytometric analysis showed that compared with the OGD-exposed cells (4 h OGD without reperfusion), the apoptosis of astrocytes was significantly increased after $12 \mathrm{~h}$ of reperfusion. However, there was no clear difference among the cells reoxygenated for 24 and $48 \mathrm{~h}$ (Fig. $1 \mathrm{~A}$ and B). Moreover, the results of MTT assay revealed that the viability of the control astrocytes was significantly elevated after $24 \mathrm{~h}$ of reperfusion while the OGD/R-exposed cells exhibited a gradual decrease in viability against the reperfusion time (Fig. 1C). In addition, $\mathrm{OGD} / \mathrm{R}$ resulted in an increase of IL- 6 , TNF- $\alpha$ and FasL in a time-dependent manner with a peak at $24 \mathrm{~h}$ of reperfusion $(\mathrm{P}<0.05)$ (Fig. 1D-F). 


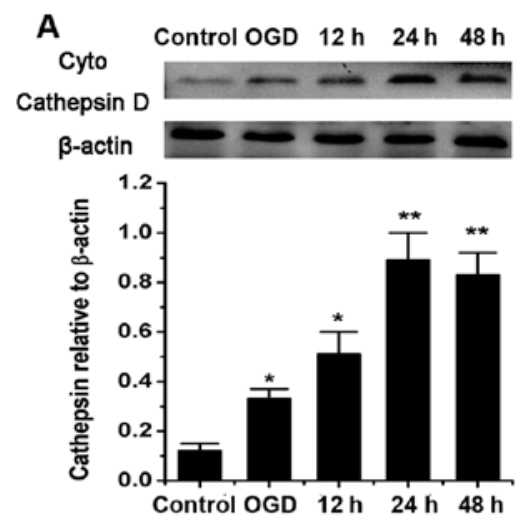

\section{B}

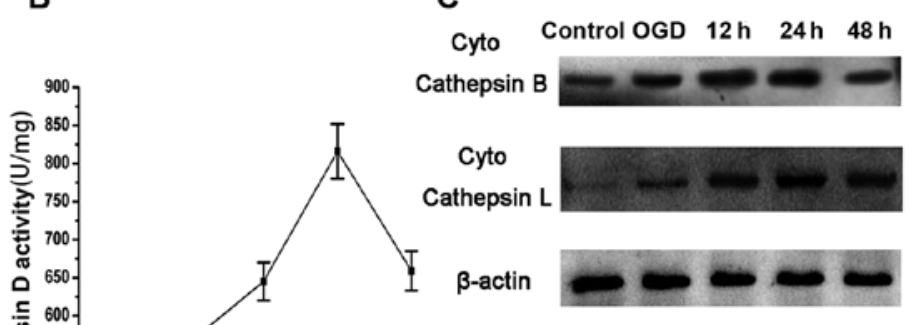

D
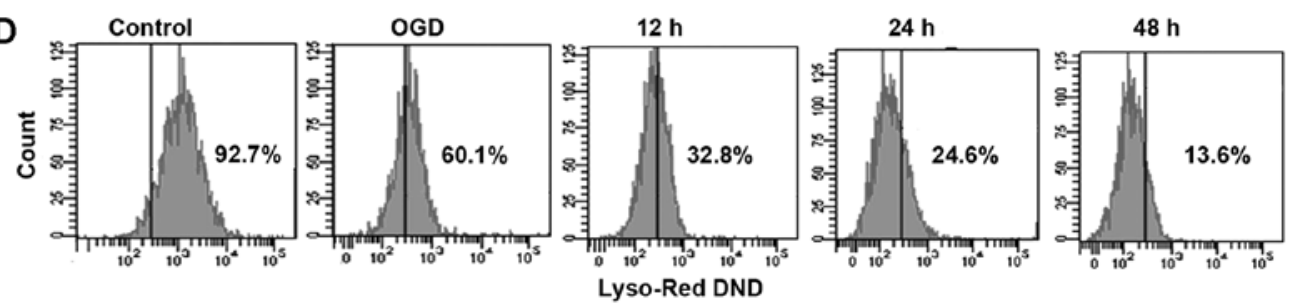

Figure 2. Oxygen-glucose deprivation/reperfusion (OGD/R) induces cathepsin $\mathrm{D}(\mathrm{Cat} \mathrm{D})$ upregulation and lysosomal dysfunction. Murine astrocytes were isolated and subjected to OGD for $4 \mathrm{~h}$ followed by reperfusion treatment over a period of time ranging from 0 to $48 \mathrm{~h}$. (A) The expression of active cytosolic (cyto) Cat D expression was determined using western blot analysis. (B) Cat D catabolic activity was assed at the indicated time points after reperfusion. (C) Western blot analysis of Cat $\mathrm{B}$ and $\mathrm{L}$ in the cytosolic fraction during OGD/R. (D) LysoTracker labeling and flow cytometric analysis of the acidic lysosome at the indicated time points after reperfusion. ${ }^{*} \mathrm{P}<0.05$, vs. control. Data are presented as the means $\pm \mathrm{SEM}$.

A

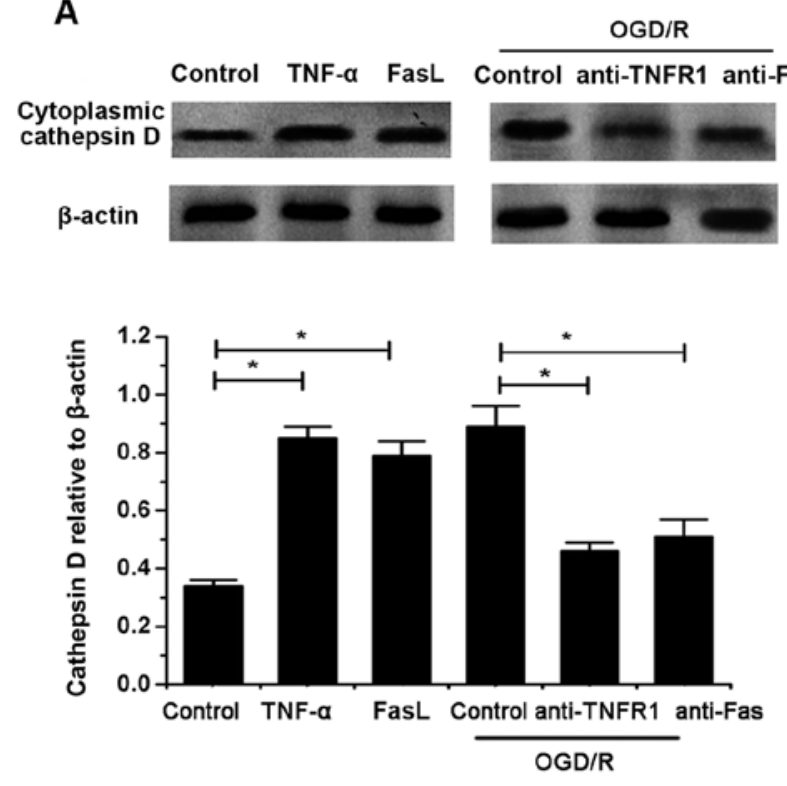

B

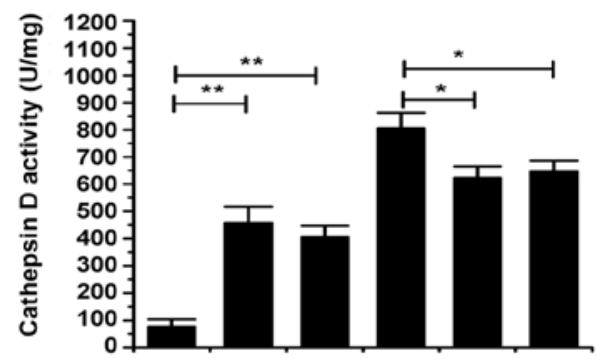

C

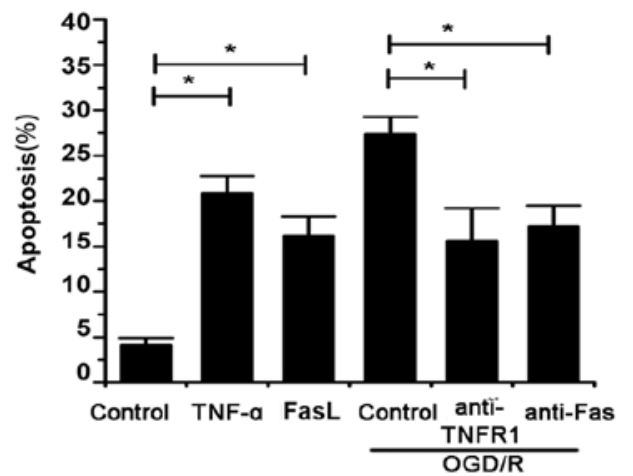

Figure 3. Tumor necrosis factor (TNF)- $\alpha$ and FasL mediate cathepsin D (Cat D) upregulation and cell apoptosis following oxygen-glucose deprivation/reperfusion (OGD/R). Changes in (A) cytosolic (cyto) Cat D levels and (B) Cat D activity in response to treatment with exogenous TNF- $\alpha$ and FasL in naïve astrocytes without OGD/R as well as to treatment with antibodies against tumor necrosis factor receptor 1 (TNFR1) and Fas in astrocytes after $24 \mathrm{~h}$ of reperfusion. (C) The apoptosis of astrocytes in response to the treatment with exogenous TNF- $\alpha$ and FasL and antibodies against TNFR1 and Fas. $\mathrm{n}=3$, ${ }^{*} \mathrm{P}<0.05$. Data are presented as the means \pm SEM.

$O G D / R$ induces Cat $D$ upregulation and lysosomal dysfunction. As shown in Fig.2A and B, the active cytosolic levels and the activity of Cat D significantly increased after $12 \mathrm{~h}$ of reperfusion and peaked at $24 \mathrm{~h}$ following the reoxygenation compared with the control cells. Furthermore, we examined other Cat proteases and the change in acidic conditions in lysosomes at various time points after OGD/R. The results showed that $\mathrm{Cat} \mathrm{B}$ and $\mathrm{L}$ were also released from lysosomes in a similar manner to Cat D (Fig. 2C). Moreover, OGD/R disturbed the innate acidic conditions of lysosomes in a time-dependent manner and this was illustrated by LysoTracker labeling (Fig. 2D). 

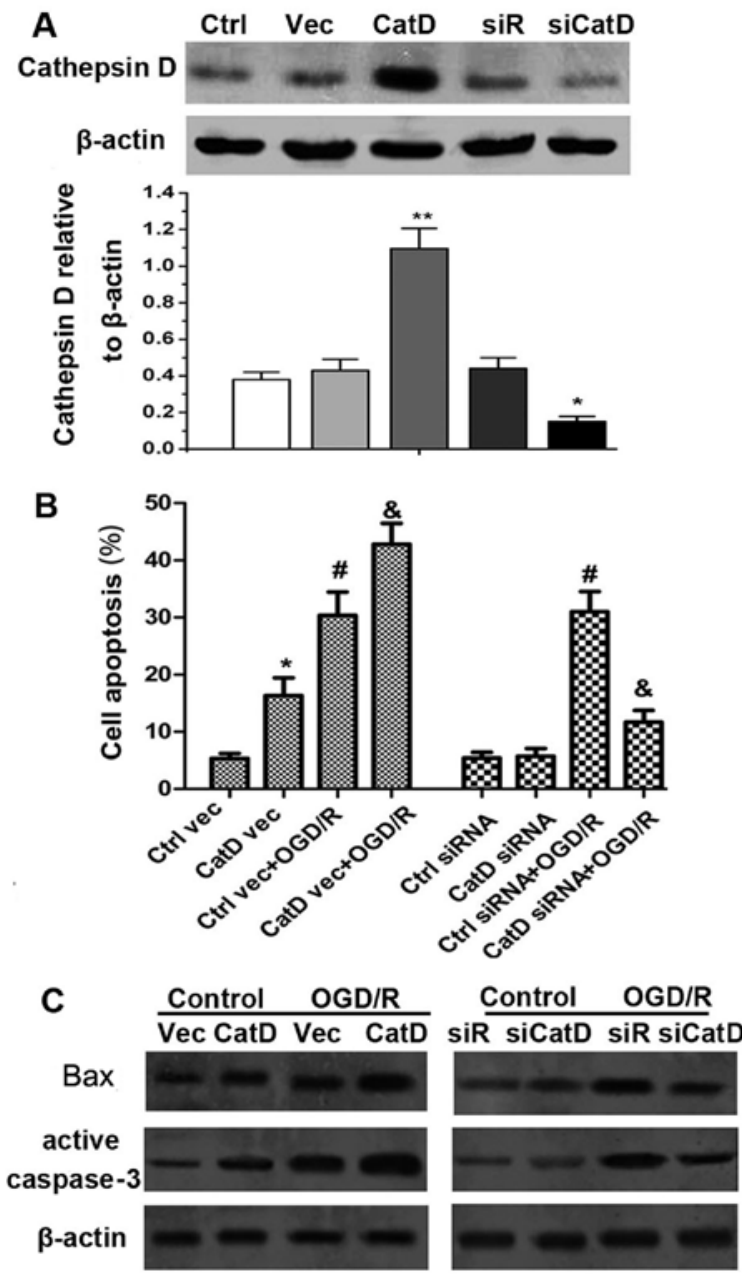

Figure 4. Cathepsin D (Cat D) mediates the apoptosis of astrocytes in a caspase-dependent manner. (A) Astrocytes were transfected with control vectors and Cat D-expressing vectors as well as control siRNA and Cat D siRNA Overall Cat D expression was determined and quantified using western blot analysis. (B) The transfected cells were treated with or without oxygen-glucose deprivation/reperfusion $(\mathrm{OGD} / \mathrm{R})$ and then cell apoptosis was examined. (C) Western blot analysis of the levels of Bax and activated caspase-3 after Cat D silencing or overexpression. $n=3,{ }^{*} \mathrm{P}<0.05$ vs. control vec/siRNA, ${ }^{\#} \mathrm{P}<0.05$, vs. CatD vec/siRNA and ${ }^{\circledR} \mathrm{P}<0.05$ vs. control vec/siRNA + OGD/R Vec, control plasmid; CatD vec, Cat D-overexpressing plasmid; siR, control siRNA; siCatD, Cat D siRNA.

TNF- $\alpha$ and FasL mediate Cat D upregulation and cell apoptosis. It was shown that TNF- $\alpha$ and FasL significantly increased the cytosolic level and activity of Cat D in astrocytes without OGD/R exposure $(\mathrm{P}<0.05)$. Moreover, we identified that the inhibition of TNFR1 and Fas using specific antibodies, markedly reversed the OGD/R-induced changes in Cat D levels and activity $(\mathrm{P}<0.05)$ (Fig. 3A and $\mathrm{B})$. Moreover, cell apoptosis was evaluated with or without $\mathrm{OGD} / \mathrm{R}$ injury in the presence and absence of TNF- $\alpha$ and FasL as well as the antibodies against TNFR1 and Fas. We found that both TNF- $\alpha$ and FasL significantly increased the apoptosis of naïve cells whereas TNFR1 and Fas blocking antibodies inhibited the cell death induced by $\mathrm{OGD} / \mathrm{R}(\mathrm{P}<0.05)$ (Fig. 3C).

OGD/R-induced cell death associated with Cat D is caspase-dependent. Cat D-overexpressing vector and siRNA were created and introduced into astrocytes and the overall expression of mature Cat D was examined as shown in Fig. 4A. After $24 \mathrm{~h}$ of reperfusion, cell death was evaluated. It was shown that Cat D overexpression enhanced cell apoptosis whereas Cat D inhibition inhibited this effect with OGD/R exposure $(\mathrm{P}<0.05)$ (Fig. 4B). In order to identify the underlying mechanism responsible for Cat D-mediated cell death, we examined the levels of apoptogenic proteins, namely Bax and caspase-3. The results showed that after $24 \mathrm{~h}$ of reperfusion, Cat $\mathrm{D}$ overexpression significantly increased the expression of Bax and caspase-3 in naïve cells and following OGD/R injury, whereas Cat D silencing reduced the expression of Bax and caspase-3 (Fig. 4C).

Cat $D$ upregulation disrupts $\Delta \Psi_{m}$ and induces ROS production. $\Delta \Psi_{\mathrm{m}}$ disturbance is an early feature of apoptosis which is indicative of mitochondrial dysfunction and the loss of membrane integrity. Cat D overexpression resulted in a profound decrease in $\Delta \Psi_{\mathrm{m}}$ following reperfusion at $24 \mathrm{~h}$ compared with the control. This was accompanied by a significant increase in the production of ROS, and this decrease lasted for $24 \mathrm{~h}$. Simultaneously, Cat D silencing markedly reversed these effects $(\mathrm{P}<0.05)$ (Fig. 5A and $\mathrm{B})$.

\section{Discussion}

Preventing astrocytes from I/R-induced damage is critical for maintaining the survival and function of neurons. In the current study, we demonstrated that Cat D expression after OGD/R contributes to the apoptosis of astrocytes and that elevated levels of TNF- $\alpha$ and FasL reinforced the expression and activity of Cat D. Moreover, Cat D disrupted $\Delta \Psi_{\mathrm{m}}$ and induced ROS production, which was accompanied by the upregulation of apoptogenic proteins, namely Bax and caspase-3.

Lysosomal enzymes, including Cats and some lipid hydrolases, play key roles in the digestion of substrates, antigen processing and extracellular matrix degradation. Research has focused on the roles of these enzymes in the initiation of apoptosis (15). When secreted following the rupture of the lysosomal membrane, Cats and lipid hydrolases may be harmful to the cellular environment, and result in pathological destruction of cellular structures (16). It has been demonstrated that the permeabilization of lysosomal membranes, and the resultant leakage of proteases play important roles in ischemic brain damage, and the leakage of lysosomal proteins was noted as an early event in the progression of brain damage (17). Cats are representative lysosomal proteases involved in ischemic astrocyte injury and their roles in cell apoptosis and necrosis have been well documented (18). A convincing study in rat models of permanent middle cerebral artery occlusion (pMCAO) showed that Cat B and $\mathrm{L}$ activation after brain ischemia triggered mitochondrial tBid-mediated astrocyte death (11). Moreover, Zhang and $\mathrm{Li}$ reported that Cat B activated the Rho kinase-JNK pathway to initiate cell apoptosis (19). Cat D is a lysosomal aspartic proteinase which plays roles in the degradation of proteins and in apoptotic processes induced by oxidative stress, cytokines, and aging (20). Conus et al reported that Cat D is involved in initiating the apoptosis of neutrophils during the resolution of inflammation (21). In addition, it has been demonstrated that Cat D overexpression in cancer cells enhances apoptosis-dependent chemosensitivity (22). 


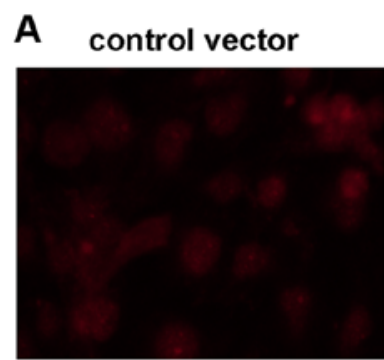

B

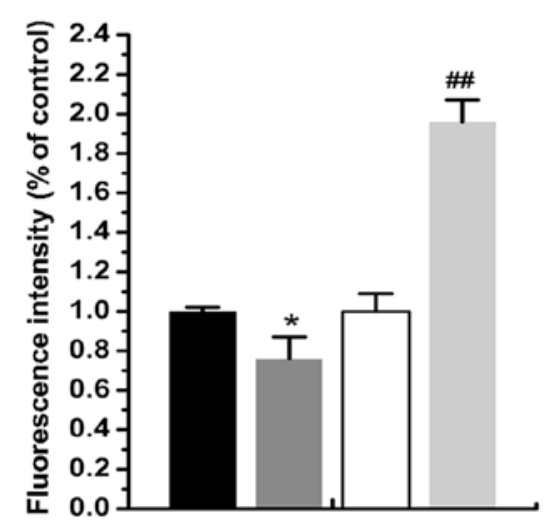

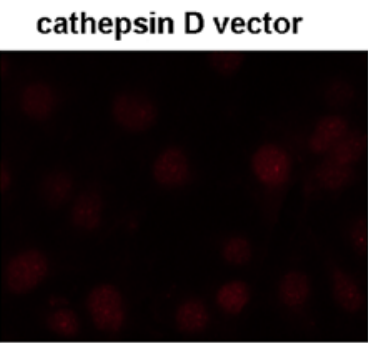

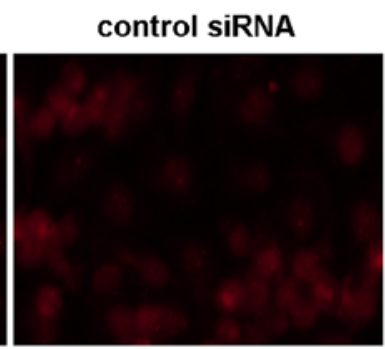

cathepsin D siRNA

C

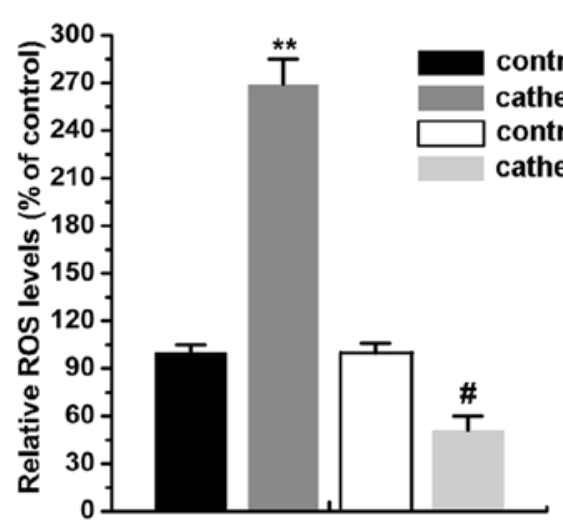

Figure 5. Cathepsin D (Cat D) upregulation impairs mitochondrial function. (A) Mitochondrial membrane staining by JC-1 in response to changes in Cat D expression following oxygen-glucose deprivation/reperfusion (OGD/R) (magnification, x200). (B) Quantification of alterations in mitochondrial membrane potential $\left(\Delta \Psi_{\mathrm{m}}\right)$ in response to changes in Cat D expression after OGD/R. (C) ROS production in Cat D-overexpressing and silencing astrocytes following $\mathrm{OGD} / \mathrm{R}$. Data are represented as the means $\pm \mathrm{SEM}$. ${ }^{*} \mathrm{P}<0.05$, vs.control vector. ${ }^{\#} \mathrm{P}<0.05,{ }^{\# \#} \mathrm{P}<0.01$, vs. control siRNA.

Therefore, lysosomal membrane permeabilization, the leakage of Cats and the subsequent apoptogenic signals contribute to I/R-induced cell death. In our study, following OGD/R injury, both cytoplasmic Cat D levels and the enzymatic activity of Cat D markedly increased with the duration of reperfusion, and this was accompanied by the increased production of inflammatory factors, namely TNF- $\alpha$, FasL and IL-6. A convincing study has demonstrated that Cat D inhibition by specific siRNA protected HeLa cells from interferon- $\gamma(\mathrm{IFN}-\gamma)$ and Fas/APO-1-induced death (23). Moreover, Heinrich et al reported that Cat D mediated the TNF-induced apoptosis of fibroblasts (24). We also found that blocking TNFR1 and Fas suppressed the levels of cytosolic Cat D and the apoptosis of astrocytes following OGD/R injury, indicating that the inflammatory factors induced by $\mathrm{OGD} / \mathrm{R}$ are responsible for the lysosome leakage and the subsequent cell apoptosis.

It has been proved that excessive ROS production and oxidative stress overloading cause lysosomal destabilization and membrane rupture, which in turn lead to propagation of apoptosis when the lysosomal contents are released into the cytosol (25). The crosstalk between lysosomes and mitochondria in cell apoptosis has attracted increasing attention over the last ten years. The release of lysosomal hydrolases may cause mitochondrial damage through the activation of phospholipases or pro-apoptotic proteins directly or indirectly. Simultaneously, lysosomal rupture appears to be a consequence of a transient oxidative stress of mitochondrial origin that follows the attack by lysosomal hydrolases and/or phospholipases, creating an amplifying loop system (26). It has been found that Cat D activates Bax in T cells and is involved in the release of cytochrome $c$ from mitochondria in fibroblasts (27). In this study, we found that Cat D upregulation induced mitochondrial membrane damage and the excessive production of ROS. Moreover, the expression of apoptogenic proteins, namely Bax and caspase-3, increased following Cat D overexpression. Liu et al reported that lysosome Cat D exocytosis from glioma cells facilitated their migration and invasion (28). However, whether lysosome Cat D is transported outside of the astrocytes following OGD/R as well as the roles of Cat $\mathrm{D}$ exocytosis in the crosstalk among glia and neurons following I/R injury remain poorly understood. Therefore, elucidating the roles of Cat D in mediating I/R damage warrants further investigation.

In conclusion, our study showed that Cat D participated in the OGD/R-induced damage to astrocytes. OGD/R induced elevated cell apoptosis and the excessive production of inflammatory cytokines, namely TNF- $\alpha$, IL- 6 and FasL, which contributed to the leakage of Cat D from the lysosome. Moreover, Cat D overexpression exacerbated OGD/R-triggered disturbances in $\Delta \Psi_{\mathrm{m}}$, ROS production and the expression of apoptogenic proteins, including Bax and caspase-3.

\section{References}

1. Tu Q, Cao H, Zhong W, Ding B and Tang X: Atorvastatin protects against cerebral ischemia/reperfusion injury through anti-inflammatory and antioxidant effects. Neural Regen Res 9: 268-275, 2014.

2. Luo Y, He Q, Kuang G, Jiang Q and Yang J: PPAR-alpha and PPAR-beta expression changes in the hippocampus of rats undergoing global cerebral ischemia/reperfusion due to PPAR-gamma status. Behav Brain Funct 10: 21, 2014.

3. Sun BZ, Chen L, Wu Q, Wang HL, Wei XB, Xiang YX and Zhang XM: Suppression of inflammatory response by flurbiprofen following focal cerebral ischemia involves the NF- $\mathrm{BB}$ signaling pathway. Int J Clin Exp Med 7: 3087-3095, 2014. 
4. Almeida AS, Queiroga CS, Sousa MF, Alves PM and Vieira HL: Carbon monoxide modulates apoptosis by reinforcing oxidative metabolism in astrocytes: role of Bcl-2. J Biol Chem 287: 10761-10770, 2012.

5. Hong S, Son MR, Yun K, Lee WT, Park KA and Lee JE: Retroviral expression of human arginine decarboxylase reduces oxidative stress injury in mouse cortical astrocytes. BMC Neurosci 15: 99, 2014

6. Sun J, Zhu Y, Zhang L and Ma Y: Effects of xuelian injection on cerebral TNF- $\alpha$, IL-1 $\beta$ and MMP-9 in rats experienced focal cerebral ischemia/reperfusion. Int J Clin Exp Med 7: 2632-2638, 2014.

7. Zhang X, Yan H, Yuan Y, Gao J, Shen Z, Cheng Y, Shen Y, Wang RR, Wang X, Hu WW, et al: Cerebral ischemia-reperfusion-induced autophagy protects against neuronal injury by mitochondrial clearance. Autophagy 9: 1321-1333, 2013.

8. Zhou J, Tan SH, Nicolas V, Bauvy C, Yang ND, Zhang J, Xue Y, Codogno P and Shen HM: Activation of lysosomal function in the course of autophagy via mTORC1 suppression and autophagosome-lysosome fusion. Cell Res 23: 508-523, 2013.

9. Qin AP, Zhang HL and Qin ZH: Mechanisms of lysosomal proteases participating in cerebral ischemia-induced neuronal death. Neurosci Bull 24: 117-123, 2008.

10. Qin AP, Liu CF, Qin YY, Hong LZ, Xu M, Yang L, Liu J, Qin ZH and Zhang HL: Autophagy was activated in injured astrocytes and mildly decreased cell survival following glucose and oxygen deprivation and focal cerebral ischemia. Autophagy 6: 738-753, 2010.

11. Xu M, Yang L, Rong JG, Ni Y, Gu WW, Luo Y, Ishidoh K, Katunuma N, Li ZS and Zhang HL: Inhibition of cysteine cathepsin B and L activation in astrocytes contributes to neuroprotection against cerebral ischemia via blocking the tBid-mitochondrial apoptotic signaling pathway. Glia 62 855-880, 2014.

12. Windelborn JA and Lipton P: Lysosomal release of cathepsins causes ischemic damage in the rat hippocampal slice and depends on NMDA-mediated calcium influx, arachidonic acid metabolism, and free radical production. J Neurochem 106: 56-69, 2008.

13. García Samartino C, Delpino MV, Pott Godoy C, Di Genaro MS, Pasquevich KA, Zwerdling A, Barrionuevo P, Mathieu P, Cassataro J, Pitossi F, et al: Brucella abortus induces the secretion of proinflammatory mediators from glial cells leading to astrocyte apoptosis. Am J Pathol 176: 1323-1338, 2010.

14. Li CY, Li X, Liu SF, Qu WS, Wang W and Tian DS: Inhibition of mTOR pathway restrains astrocyte proliferation, migration and production of inflammatory mediators after oxygen-glucose deprivation and reoxygenation. Neurochem Int 83-84: 9-18, 2015.

15. Cesen MH, Pegan K, Spes A and Turk B: Lysosomal pathways to cell death and their therapeutic applications. Exp Cell Res 318: $1245-1251,2012$.
16. Lee SJ, Park MH, Kim HJ and Koh JY: Metallothionein-3 regulates lysosomal function in cultured astrocytes under both normal and oxidative conditions. Glia 58: 1186-1196, 2010.

17. Lipton P: Lysosomal membrane permeabilization as a key player in brain ischemic cell death: a 'lysosomocentric' hypothesis for ischemic brain damage. Transl Stroke Res 4: 672-684, 2013.

18. Yamashima $\mathrm{T}$ and Oikawa S: The role of lysosomal rupture in neuronal death. Prog Neurobiol 89: 343-358, 2009.

19. Zhang ZB and Li ZG: Cathepsin B and phospo-JNK in relation to ongoing apoptosis after transient focal cerebral ischemia in the rat. Neurochem Res 37: 948-957, 2012.

20. Haendeler J, Popp R, Goy C, Tischler V, Zeiher AM and Dimmeler S: Cathepsin D and $\mathrm{H}_{2} \mathrm{O}_{2}$ stimulate degradation of thioredoxin-1: implication for endothelial cell apoptosis. J Biol Chem 280: 42945-42951, 2005.

21. Conus S, Perozzo R, Reinheckel T, Peters C, Scapozza L, Yousefi S and Simon HU: Caspase- 8 is activated by cathepsin D initiating neutrophil apoptosis during the resolution of inflammation. J Exp Med 205: 685-698, 2008.

22. Beaujouin M and Liaudet-Coopman E: Cathepsin D overexpressed by cancer cells can enhance apoptosis-dependent chemo-sensitivity independently of its catalytic activity. In: Hormonal Carcinogenesis V. Li JJ, Li S, Mohla S, Rochefort H and Maudelonde T (eds). Vol 617. Springer, New York, NY, pp453-461, 2008.

23. Deiss LP, Galinka H, Berissi H, Cohen O and Kimchi A: Cathepsin D protease mediates programmed cell death induced by interferon-gamma, Fas/APO-1 and TNF-alpha. EMBO J 15: 3861-3870, 1996.

24. Heinrich M, Neumeyer J, Jakob M, Hallas C, Tchikov V, Winoto-Morbach S, Wickel M, Schneider-Brachert W, Trauzold A, Hethke A, et al: Cathepsin D links TNF-induced acid sphingomyelinase to Bid-mediated caspase- 9 and -3 activation. Cell Death Differ 11: 550-563, 2004

25. Repnik U and Turk B: Lysosomal-mitochondrial cross-talk during cell death. Mitochondrion 10: 662-669, 2010.

26. Terman A, Gustafsson B and Brunk UT: The lysosomal-mitochondrial axis theory of postmitotic aging and cell death. Chem Biol Interact 163: 29-37, 2006.

27. Bidère N, Lorenzo HK, Carmona S, Laforge M, Harper F, Dumont $\mathrm{C}$ and Senik A: Cathepsin D triggers Bax activation, resulting in selective apoptosis-inducing factor (AIF) relocation in $\mathrm{T}$ lymphocytes entering the early commitment phase to apoptosis. J Biol Chem 278: 31401-31411, 2003.

28. Liu Y, Zhou Y and Zhu K: Inhibition of glioma cell lysosome exocytosis inhibits glioma invasion. PLoS One 7: e45910, 2012. 